\title{
Precise Cost Analysis via Local Reasoning
}

\author{
Diego E. Alonso-Blas, Puri Arenas, and Samir Genaim \\ DSIC, Complutense University of Madrid (UCM), Spain
}

\begin{abstract}
The classical approach to static cost analysis is based on first transforming a given program into a set of cost relations, and then solving them into closed-form upper-bounds. The quality of the upper-bounds and the scalability of such cost analysis highly depend on the precision and efficiency of the solving phase. Several techniques for solving cost relations exist, some are efficient but not precise enough, and some are very precise but do not scale to large cost relations. In this paper we explore the gap between these techniques, seeking for ones that are both precise and efficient. In particular, we propose a novel technique that first splits the cost relation into several atomic ones, and then uses precise local reasoning for some and less precise but efficient reasoning for others. For the precise local reasoning, we propose several methods that define the cost as a solution of a universally quantified formula. Preliminary experiments demonstrate the effectiveness of our approach.
\end{abstract}

\section{Introduction}

Static Cost analysis (a.k.a. resource usage analysis) aims at statically determining the amount of resources (e.g., memory, execution steps, etc.) required to execute a given program safely, i.e., without running out of resources. Applications of cost analysis range from detecting performance bottlenecks at the development stage, to providing resource consumption guarantees at runtime.

Several cost analysis frameworks exist 4131510. Although different in their underlying techniques, they all report the cost as a closed-form upper-bound function ( $U B$ for short) in terms of the input parameters. This paper uses the classical approach of Wegbreit [18, in particular its extension for JAVA bytecode 4, where the analysis is carried out in two phases: (1) the input program is transformed into a set of cost relations ( $C R s$ for short) that define its cost; and (2) the CRs are solved into UBs. While the first phase depends on the programming language in which the program is written [41112917, the second phase is common to all analyses that are based on this approach. In this paper we focus on the second phase, i.e., on developing techniques for solving CRs. However, we provide enough details to clarify how $C R s$ are related to programs.

Example 1. The JAVA class depicted in Fig.11implements a dynamic array, where field data is used to store its elements, and field size represents the number of such elements. Method add adds the elements of the array elems to the dynamic array. When the array data is full (L6), it is replaced by a new one of double 


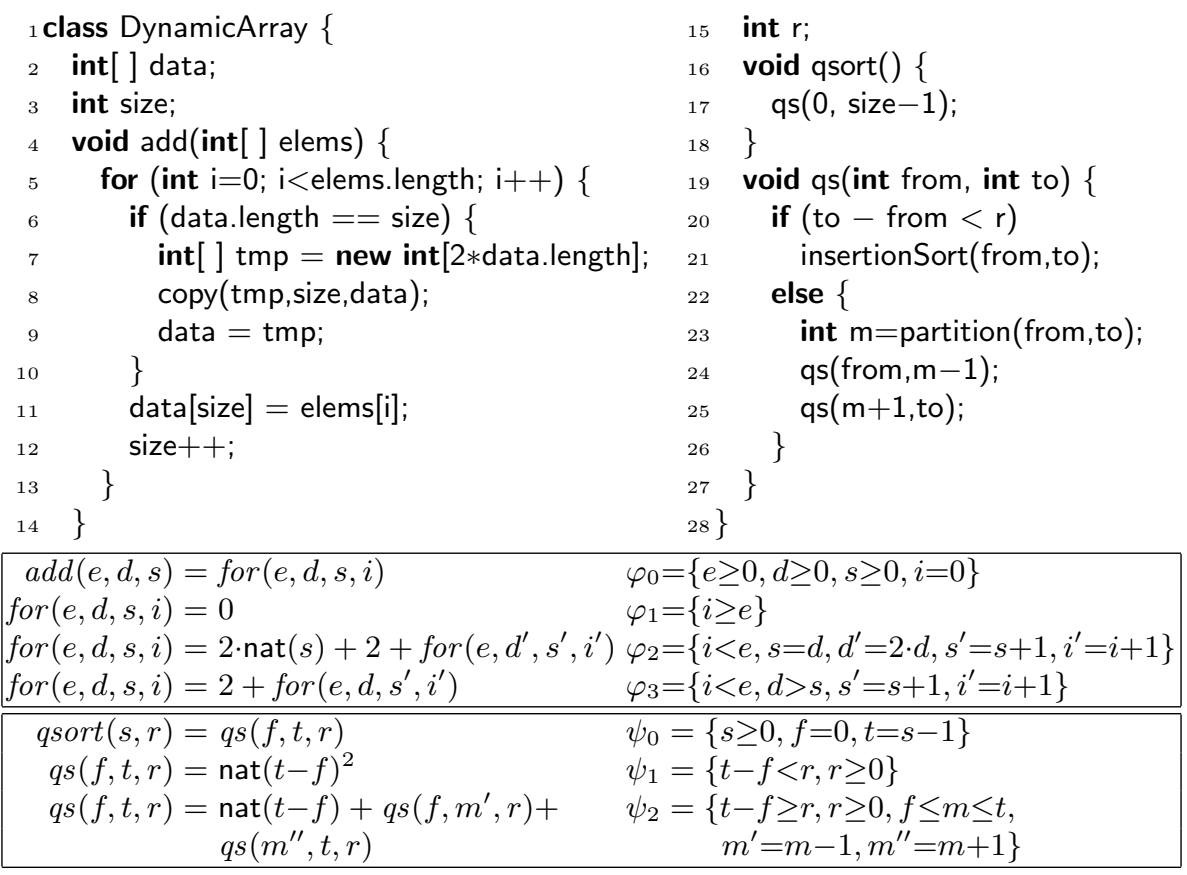

Fig. 1. Above, JAvA code of a DynamicArray class. Below, the $C R s$ of the methods

size (L7 79 ). Methods qsort and qs sort the array using a variation of Quick Sort, which resorts to Insertion Sort when the segment to be sorted is shorter than a threshold defined by field $r$. Methods copy, partition, and insertionSort are omitted.

Below the JAVA code we show the corresponding $C R s$, generated using a cost model that counts array accesses. Let us explain the $C R$ of method add. Variables $e, d, s$, and $i$ stand for the lengths of arrays elems and data and the values of size and i. Expression nat $(e)$ is an abbreviation for $\max \{e, 0\}$. The first equation states that the cost of $a d d(e, d, s)$ is as that of for $(e, d, s, i)$. The constraints on the right impose conditions and relations on the variables. The second equation is for the case of exiting the loop $(i \geq e)$. The third one is for the case in which the array is resized. In such case the cost is $2 \cdot$ nat $(s)$ (the cost assumed for copy), plus 2 (the accesses at $\mathrm{L} 11$ ), plus the cost of the remaining iterations for $\left(e, d^{\prime}, s^{\prime}, i^{\prime}\right)$. Note that $d^{\prime}=2 \cdot d$ states that the size of array data is doubled. The fourth equation describes the case in which the array is not resized. The equations of qsort are defined similarly. We note that nat $(t-f)^{2}$ and nat $(t-f)$ correspond to the cost of insertionSort and partition respectively. The constraint $f \leq m \leq t$ in $\psi_{2}$ is an input-output summary inferred for the value m returned by method partition. Methods add and qsort, respectively, have linear and quadratic worst-case complexity.

Early works on cost analysis [119] relied on Computer Algebra Systems (CAS) for solving CRs. They can only handle cases in which the CRs can be 
transformed into recurrence equations (the only valid input for CAS). This, however, is a very limited subset because $C R s$ allow using constraints to define complex applicability conditions and relations between the variables. To overcome this limitation, recent works [36 have developed dedicated tools for solving CRs into UBs. They are mostly based on the use of program analysis techniques. These works are our starting point.

The techniques of [3] are based on assuming worst-case behaviour for all loop iterations. It is very efficient and can handle a wide class of $C R s$. To solve the $C R$ for, this technique infers that $2+2 \cdot \operatorname{nat}(e+s-1)$ is an $U B$ on the cost of any iteration of for, and it infers that there is at most nat $(e-i)$ iterations of for, from which it concludes that nat $(e-i) \cdot(2+2 \cdot \operatorname{nat}(e+s-1))$ is an $U B$ for $C R$ for. Note that this is a quadratic $U B$ while the actual cost is linear. In the case of qsort, the loss of precision is even bigger. It first infers that nat $(t-f)^{2}$ is an $U B$ on the cost of each call to qs, and that there are at most $2^{\text {nat }(t-f)}$ of such calls. Then, it concludes that $(t-f)^{2} \cdot 2^{\text {nat }(t-f)}$ is an $U B$ for $C R q s$, while the actual cost is quadratic.

The above imprecision issue, among others, was addressed in [6] where precise and novel techniques for solving CRs were proposed. They are based on defining the cost as a solution of a corresponding first-order universally quantified formula. This method, as expected, would obtain the most precise UBs for the CRs for and $q s$, however, it has two major limitations: (1) a template $U B$ has to be provided by the user; and (2) the use of a quantifier elimination procedure for real numbers renders the technique impractical.

In this paper we explore the gap between [3] and [6], seeking for solving techniques with efficiency close to [3] and precision close to [6]. Concretely, we develop a novel technique that breaks down the input $C R$ into atomic $C R s$ of simpler form, solves each of them separately, and then combines the results into an $U B$ for the original $C R$. Our main observation is that it is enough to solve few atomic CRs precisely, while solving the others as in [3, without affecting the overall precision. We also propose several methods for precisely solving atomic $C R s$, which are based on the idea of specifying the cost using universally quantified formulas as in 6]. However, we do not require the user to provide any template, and, importantly, the generated formulas have almost a linear form for which quantifier elimination can be done efficiently. Our prototype implementation and experiments [1] demonstrate the effectiveness of this approach.

This paper is organised as follows. Sec. 2 provides the required background on CRs. Sec. 3 is the technical core of the paper. Sec. 4 describes a prototype implementation and preliminary experiments. Finally, in Sec. 5 we conclude and discuss related work.

\section{Cost Relations: Syntax and Semantics}

In this section we recall some basic notions related to $C R s[3$. The sets of real, rational, and integer values are denoted by $\mathbb{R}, \mathbb{Q}$, and $\mathbb{Z}$, respectively. $\mathbb{R}^{+}, \mathbb{Q}^{+}$, and $\mathbb{Z}^{+}$denote their non-negative subsets. Variables are denoted by $x, y, z$, and 
$w$, possibly subscripted. Values from $\mathbb{R}, \mathbb{Q}$, and $\mathbb{Z}$ are denoted, respectively, by $r, q$, and $v$. A sequence of elements of type $t$ is denoted by $\bar{t}$. The set of variables of $t$ is denoted by vars $(t)$. An assignment $\sigma: \mathcal{V} \mapsto \mathcal{D}$ maps variables from $\mathcal{V}$ to values from $\mathcal{D}$ and $\sigma(\bar{t})$ denotes the replacement of any $x \in \operatorname{vars}(\bar{t})$ by $\sigma(x)$.

A linear expression has the form $q_{0}+q_{1} \cdot x_{1}+\cdots+q_{n} \cdot x_{n}$. A linear constraint has the form $l_{1} \leq l_{2}, l_{1}=l_{2}$, or $l_{1} \geq l_{2}$, where $l_{1}$ and $l_{2}$ are linear expressions and $\operatorname{vars}\left(l_{1}\right) \cup \operatorname{vars}\left(l_{2}\right) \subseteq \mathbb{Z}$. The constraints $l_{1}>l_{2}$ and $l_{1}<l_{2}$ abbreviate $l_{1} \geq l_{2}+1$ and $l_{1}+1 \leq l_{2}$, respectively. We use $\varphi, \phi$, and $\psi$, possibly subscripted, to denote conjunctions (often written as sets) of linear constraints. We say that $\varphi$ is satisfiable if there is an assignment $\sigma$ for $\operatorname{vars}(\varphi)$ such that $\sigma(\varphi)$ is true, denoted as $\sigma \models \varphi$. If $\sigma \models \varphi$ for every assignment $\sigma$ for $\operatorname{vars}(\varphi)$ then $\varphi$ is a valid formula.

Definition 1 (cost expression). A cost expression $e$ is defined as:

$$
e::=q|\operatorname{nat}(l)| \log _{a}(1+\operatorname{nat}(l))\left|a^{\text {nat }(l)}-1\right| e+e \mid e \cdot e
$$

where $q \in \mathbb{Q}^{+}$, nat $(l)=\max \{l, 0\}, a>1 \in \mathbb{Z}^{+}$, and $l$ is a linear expression.

Note that we use $a^{\text {nat }(l)}-1$, instead of simply $a^{\text {nat }(l)}$, for the sake of simplifying the formal presentation (we explain this after Lemma 3 ).

Definition 2 (cost relation). A cost relation is a set of cost equations of the form $\left\langle C(\bar{x})=e+\sum_{j=1}^{k} D_{j}\left(\bar{y}_{j}\right), \varphi\right\rangle$, where $C$ and $D_{j}$ are cost relation symbols.

Intuitively, a cost equation $\left\langle C(\bar{x})=e+\sum_{j=1}^{k} D_{j}\left(\bar{y}_{j}\right), \varphi\right\rangle$ states that the cost of $C(\bar{x})$ is $e$ plus the sum of the costs of $D_{1}\left(\bar{y}_{1}\right), \ldots, D_{k}\left(\bar{y}_{k}\right)$. The linear constraint $\varphi$ specifies the values of $\bar{x}$ for which the equation is applicable, and defines relations among the different variables. Since $C R s$ usually originate from programs, it is often helpful to think of each $C R$ symbol as a (non-deterministic) procedure, in which case we say that $C$ calls $D_{1}, \ldots, D_{k}$.

Without loss of generality, in what follows we assume that the input $C R$ includes a single $C R$ symbol. Namely, in Def. 2 we have $D_{j}=C$. We call such $C R s$ stand-alone. To handle $C R s$ with more than one $C R$ symbol, we rely on the compositional approach of 3 ] which we briefly explain next. In a first step, the input $C R$ is transformed into a form in which all recursions are direct, i.e. an equation that defines $C$ can either call itself directly, or other $C R$ symbols that do not call $C$ (directly or indirectly). In a second step, the $C R s$ are solved iteratively, where in each iteration we solve those that do not depend on any other symbols (there must be at least one), and then substitute the result in the calling contexts. In the rest of the paper $C R$ refers to a stand-alone $C R$.

To define the cost assigned by $C$ to a concrete input $\bar{v}$, we use evaluation trees. A (possibly infinite) tree will be denoted by node $\left(r,\left\langle T_{1}, \ldots, T_{k}\right\rangle\right)$, where $r \in \mathbb{R}^{+}$is the value of the root and $T_{1}, \ldots, T_{k}$ are sub-trees.

Definition 3 (evaluation tree). Given $a \mathrm{CR} C$ and an input $\bar{v}$, we say that node $\left(r,\left\langle T_{1}, \ldots, T_{k}\right\rangle\right)$ is an evaluation tree for $C(\bar{v})$ iff there exists an equation $\mathcal{E} \equiv\left\langle C(\bar{x})=e+\sum_{j=1}^{k} C\left(\bar{y}_{j}\right), \varphi\right\rangle$ and $\sigma: \operatorname{vars}(\mathcal{E}) \mapsto \mathbb{Z}$ such that: (1) $\sigma\left(x_{i}\right)=v_{i}$ and $\sigma \models \varphi$; (2) $r=\sigma(e)$; and (3) each $T_{i}$ is an evaluation tree for $C\left(\sigma\left(\bar{y}_{i}\right)\right)$. 
Intuitively, when viewing $C$ as a procedure, an evaluation tree can be seen as a recursion tree where the call $C(\bar{v})$ is evaluated as follows: we pick an equation that defines $C$ and an assignment $\sigma$ that satisfies the equation's constraints; we evaluate $\sigma(e)$ into $r$, and we recursively call each $C\left(\sigma\left(\bar{y}_{i}\right)\right)$. Note that an evaluation tree can be infinite. Note also that $C(\bar{v})$ might have several evaluation trees, due to the nondeterminism induced by choosing an equation for $C$ and a satisfying assignment $\sigma$ for $\varphi$. The set of all evaluation trees for $C(\bar{v})$ is denoted by Trees $(C(\bar{v}))$. The set of all possible costs for $C(\bar{v})$ is then defined as Answers $(C(\bar{v}))=\{\operatorname{Sum}(T) \mid T \in \operatorname{Trees}(C(\bar{v}))\}$, where $\operatorname{Sum}(T)$ is the sum of all nodes of $T$. Our interest is to approximate $C R s$ by mean of closed-form $U B s$ functions, i.e., functions of the form $f(\bar{x})=e$, where $\operatorname{vars}(e) \subseteq \bar{x}$.

Definition 4 (upper bound). A function $C^{+}: \mathbb{Z}^{n} \mapsto \mathbb{R}^{+}$is an UB for a CR $C$, iff for any input $\bar{v} \in \mathbb{Z}^{n}$ and cost $r \in \operatorname{Answers}(C(\bar{v}))$ we have $C^{+}(\bar{v}) \geq r$.

Next we overview the approach of 3 for solving a $C R$ into an $U B$. Suppose we have two functions $h(\bar{x})=e_{1}$ and $g(\bar{x})=e_{2}$, where $e_{1}$ and $e_{2}$ are cost expressions, such that for any $T \in \operatorname{Trees}(C(\bar{v}))$ the following holds (i) $h(\bar{v})$ is an $U B$ on the depth of $T$; and (ii) $g(\bar{v})$ is an $U B$ on the value of any node of $T$. Now assuming that $d$ is the maximum number of recursive calls in any equation of $C$, i.e., the maximum branching factor of its evaluation trees, then $C^{+}(\bar{x})=g(\bar{x}) \cdot \mathcal{N}$ where $\mathcal{N}=h(\bar{x})$ if $d=1$, and $\mathcal{N}=d^{h(\bar{x})}$ if $d>1$. Technically, in [3], $h(\bar{x})$ is computed by inferring a linear ranking function [8] that bounds the recursion depth of $C$, and $g(\bar{x})$ is computed by relying on linear invariants.

Example 2. Consider the $C R$ for in Fig. [1. The technique of [3] infers $h(e, d, s, i)=$ nat $(e-i)$ and $g(e, d, s, i)=2+2 \cdot \operatorname{nat}(e+s-1)$. Then, since the branching factor is $d=1$, it reports the $U B f_{0 r}{ }^{+}(e, d, s, i)=\operatorname{nat}(e-i) \cdot(2+2 \cdot$ nat $(e+s-1))$. For $C R q s$, it infers $h(f, t, r)=$ nat $(t-f)$ and $g(f, t, r)=$ nat $(t-f)^{2}$. Then, since the branching factor is $d=2$, it reports the $U B q s^{+}(f, t, r)=\operatorname{nat}(t-f)^{2} \cdot 2^{\text {nat }(t-f)}$.

Maximisation procedure. We rely on the technique of 3 that generates $g(\bar{x})$ as we explain next. Let $e$ be the cost expression that is contributed by an equation of $C$, and let $b$ be a cost sub-expression of $e$. As explained in Def. 3, when generating the nodes of an evaluation tree $T \in \operatorname{Trees}(C(\bar{v}))$, we evaluate $\sigma(e)$ to $r$. This evaluation requires computing $\sigma(b)$. We call $\sigma(b)$ an instance of $b$. We reuse the techniques of [3] to infer a cost expression $\hat{b}(\bar{x})$ that satisfies the following: for any input $\bar{v}, T \in \operatorname{Trees}(C(\bar{v}))$ and any instance $\sigma(b)$ of $b$ in $T$, we have $\hat{b}(\bar{v}) \geq \sigma(b)$. Intuitively, $\hat{b}(\bar{x})$ is a function that bounds each contribution of $b$ to the total cost. We call $\hat{b}(\bar{x})$ the maximisation of $b$, and, in our implementation, we compute it reusing the components of 3 .

\section{Solving Cost Relations in Closed-Form Upper-Bounds}

In this section we present our approach for solving a $C R C$ into an $U B$. We assume that $C$ is defined by $m$ equations of the form $\left\langle C(\bar{x})=e_{i}+\sum_{j=1}^{k_{i}} C\left(\bar{y}_{i j}\right), \varphi_{i}\right\rangle$, 


\begin{tabular}{|c|c|c|}
\hline for $_{1}$ & $\left\{\begin{array}{l}\operatorname{for}(e, d, s, i)=0 \\
\operatorname{for}(e, d, s, i)=2 \cdot \operatorname{nat}(s)+f o r\left(e, d^{\prime}, s^{\prime}, i^{\prime}\right) \\
\operatorname{for}(e, d, s, i)=\text { for }\left(e, d, s^{\prime}, i^{\prime}\right)\end{array}\right.$ & $\begin{array}{l}\varphi_{1}=\{i \geq e\} \\
\varphi_{2}=\left\{i<e, s=d, d^{\prime}=2 \cdot d, s^{\prime}=s+1, i^{\prime}=i+1\right\} \\
\varphi_{3}=\left\{i<e, d>s, s^{\prime}=s+1, i^{\prime}=i+1\right\}\end{array}$ \\
\hline for $_{2}$ & $\left\{\begin{array}{l}f o r(e, d, s, i)=0 \\
f o r(e, d, s, i)=2+\text { for }\left(e, d^{\prime}, s^{\prime}, i^{\prime}\right) \\
\text { for }(e, d, s, i)=\text { for }\left(e, d, s^{\prime}, i^{\prime}\right)\end{array}\right.$ & $\begin{array}{l}\varphi_{1}=\{i \geq e\} \\
\varphi_{2}=\left\{i<e, s=d, d^{\prime}=2 \cdot d, s^{\prime}=s+1, i^{\prime}=i+1\right\} \\
\varphi_{3}=\left\{i<e, d>s, s^{\prime}=s+1, i^{\prime}=i+1\right\}\end{array}$ \\
\hline for $_{3}$ & $\begin{array}{l}f \circ r(e, d, s, i)=0 \\
f o r(e, d, s, i)=f o r\left(e, d^{\prime}, s^{\prime}, i^{\prime}\right) \\
\operatorname{for}(e, d, s, i)=2+\text { for }\left(e, d, s^{\prime}, i^{\prime}\right)\end{array}$ & $\begin{array}{l}\varphi_{1}=\{i \geq e\} \\
\varphi_{2}=\left\{i<e, s=d, d^{\prime}=2 \cdot d, s^{\prime}=s+1, i^{\prime}=i+1\right\} \\
\varphi_{3}=\left\{i<e, d>s, s^{\prime}=s+1, i^{\prime}=i+1\right\}\end{array}$ \\
\hline$q s_{1}$ & $\begin{array}{l}q s(f, t, r)=\operatorname{nat}(t-f)^{2} \\
q s(f, t, r)=q s\left(f, m^{\prime}, r\right)+q s\left(m^{\prime \prime}, t, r\right)\end{array}$ & $\begin{aligned} \psi_{1}= & \{t-f<r, r \geq 0\} \\
\psi_{2}= & \{t-f \geq r, r \geq 0, f \leq m \leq t \\
& \left.m^{\prime}=m-1, m^{\prime \prime}=m+1\right\}\end{aligned}$ \\
\hline$q s_{2}\{$ & $\begin{aligned} q s(f, t, r)= & 0 \\
q s(f, t, r)= & \operatorname{nat}(t-f)+q s\left(f, m^{\prime}, r\right)+ \\
& q s\left(m^{\prime \prime}, t, r\right)\end{aligned}$ & $\begin{aligned} \psi_{1}= & \{t-f<r, r \geq 0\} \\
\psi_{2}= & \{t-f \geq r, r \geq 0, f \leq m \leq t, \\
& \left.m^{\prime}=m-1, m^{\prime \prime}=m+1\right\}\end{aligned}$ \\
\hline
\end{tabular}

Fig. 2. The sparse $C R s$ of for and $q s$ of Fig. 1

$1 \leq i \leq m$. Our approach is presented in two steps: we reduce the problem of solving $C$ to solving atomic $C R s$, and then we focus on solving atomic CRs.

Observe that cost expressions, as in Def. 11, can be normalised into the form $P_{1}+\cdots+P_{h}$, where each $P_{i}$ is a product of cost expressions $b_{i 1}, \ldots, b_{i p_{i}}$ with $b_{i j} \in\left\{q, \operatorname{nat}(l), \log _{a}(1+\operatorname{nat}(l)), a^{\text {nat }(l)}-1\right\}$. For simplicity, since $q$ is non-negative, we assume it is given as nat $(q)$. We assume that each $e_{i}$ in $C$ is given in this form. Let $P_{C}=\left\{P_{1}, \ldots, P_{t}\right\}$ be the multiset of all non-zero product cost expressions that appear in $C$ (i.e., the products of $\left.e_{1}, \ldots, e_{m}\right)$. We define $C_{i}$ as the $C R$ obtained from $C$ by removing all $P_{j} \in P_{C}$ with $j \neq i$. Namely, in $C_{i}$ there is exactly one equation that contributes $P_{i}$, the others contribute 0 . We call such CRs sparse and the equation that includes $P_{i}$ is called the main equation.

Example 3. Consider the CRs for and qs in Fig. 1. Their products are respectively $P_{f o r}=\{2 \cdot \operatorname{nat}(s), 2,2\}$ and $P_{q s}=\{\operatorname{nat}(t-f) \cdot \operatorname{nat}(t-f)$, nat $(t-f)\}$. Their corresponding sparse $C R s$ are depicted in Fig. 2 .

Observation 1 If $C_{i}^{+}(\bar{x})$ is an $\mathrm{UB}$ for the sparse $\mathrm{CR} C_{i}$, for all $1 \leq i \leq t$, then $C^{+}(\bar{x})=C_{1}^{+}(\bar{x})+\cdots+C_{t}^{+}(\bar{x})$ is an UB for $C$.

The above observation explains how an $U B$ for $C$ can be obtained from $U B s$ for its sparse $C R s C_{1}, \ldots, C_{t}$. Thus, we can focus on solving sparse $C R s$. We first explain the idea intuitively. Assume that $b_{i 1} \cdot b_{i 2}$ is the product in the main equation of $C_{i}$. Given an arbitrary $T \in \operatorname{Trees}\left(C_{i}(\bar{v})\right)$, the cost of each of its nodes is either 0 or an instance of $b_{i 1} \cdot b_{i 2}$. Let $\sigma_{1}\left(b_{i 1} \cdot b_{i 2}\right), \ldots, \sigma_{h}\left(b_{i 1} \cdot b_{i 2}\right)$ be the instances of $b_{i 1} \cdot b_{i 2}$ in $T \in \operatorname{Trees}\left(C_{i}(\bar{v})\right)$, then the cost of $T$ is $S=\sum_{j=1}^{h} \sigma_{j}\left(b_{i 1} \cdot b_{i 2}\right)$. As explained in Sec. 2, we can compute a function $\hat{b}_{i 1}(\bar{x})$ such that $\hat{b}_{i 1}(\bar{v}) \geq \sigma_{j}\left(b_{i 1}\right)$ for each $1 \leq j \leq h$. Using $\hat{b}_{i 1}(\bar{v})$ we bound $S$ as follows: 


$$
S=\sum_{j=1}^{h} \sigma_{j}\left(b_{i 1} \cdot b_{i 2}\right) \leq \sum_{j=1}^{h} \hat{b}_{i 1}(\bar{v}) \cdot \sigma_{j}\left(b_{i 2}\right)=\hat{b}_{i 1}(\bar{v}) \cdot \sum_{j=1}^{h} \sigma_{j}\left(b_{i 2}\right)
$$

Now assume that we have a function $f^{+}(\bar{x})$ such that $f^{+}(\bar{v}) \geq \sum_{j=1}^{h} \sigma_{j}\left(b_{i 2}\right)$, then $S \leq \hat{b}_{i 1}(\bar{v}) \cdot f^{+}(\bar{v})$. Thus, since the above reasoning is done for an arbitrary $T$, we can conclude that $\hat{b}_{i 1}(\bar{x}) \cdot f^{+}(\bar{x})$ is an $U B$ for $C_{i}$. Now to compute $f^{+}(\bar{x})$, we consider a $C R C_{i 2}$ that is obtained from $C_{i}$ by replacing $b_{i 1} \cdot b_{i 2}$ by $b_{i 2}$. Clearly, $C_{i 2}(\bar{v})=\sum_{j=1}^{h} \sigma_{j}\left(b_{i 2}\right)$, and thus any $U B$ for $C_{i 2}$ defines a valid $f^{+}(\bar{x})$. This reduces the problem of solving $C_{i}$ to that of solving $C_{i 2}$, which is simpler since its main equation includes a basic cost expression. Note that, in a similar way, we could build $C_{i 1}$ using $\hat{b}_{i 2}(\bar{x})$ and then use it to find an $U B$ for $C_{i}$.

Formally, given a sparse $C R C_{i}$ with a product $b_{i 1} \cdots b_{i p_{i}}$ in its main equation, we define the atomic $C R C_{i j}$ as the one obtained from $C_{i}$ by replacing its product by $b_{i j}$ (i.e., removing all $b_{i k}$ with $k \neq j$ ).

Example 4. Consider the sparse CRs depicted in Fig. 2. The following are possible atomic $C R s$ for for $_{1}$ and $q s_{1}$

\begin{tabular}{|c|c|c|c|}
\hline \multicolumn{2}{|l|}{ for $_{12}$} & \multicolumn{2}{|l|}{$q s_{11}$} \\
\hline for $(e, d, s, i)=0$ & & $q s(f, t, r)=\operatorname{nat}(t-f)$ & $\psi_{1}$ \\
\hline $\operatorname{for}(e, d, s, i)=\operatorname{nat}(s)+f o r\left(e, d^{\prime}, s^{\prime}, i^{\prime}\right)$ & $\varphi_{2}$ & $q s(f, t, r)=q s\left(f, m^{\prime}, r\right)+q s\left(m^{\prime \prime}, t, r\right)$ & $\psi_{2}$ \\
\hline for $(e, d, s, i)=\operatorname{for}\left(e, d, s^{\prime}, i^{\prime}\right)$ & $\varphi_{3}$ & & \\
\hline
\end{tabular}

in which nat $(s)$ and nat $(t-f)$ are selected as basic cost expressions. CRs for 2 , $f r_{3}$ and $q s_{2}$ are already atomic. They correspond to $f r_{21}, f_{0} r_{31}$ and $q s_{21}$.

Lemma 1. Let $C_{i}$ be a sparse $\mathrm{CR}, b_{i 1} \cdots b_{i p_{i}}$ the product in its main equation, and $C_{i j}$ an atomic $\mathrm{CR}$ of $C_{i}$. If $C_{i j}^{+}(\bar{x})$ is an $\mathrm{UB}$ for $C_{i j}(\bar{x})$, then $C_{i}^{+}(\bar{x})=$ $C_{i j}^{+}(\bar{x}) \cdot \prod_{k \neq j} \hat{b}_{i k}(\bar{x})$ is an UB for $C_{i}$.

The above lemma allows focusing on finding an $U B$ for a single atomic $C_{i j}$ and then combine the result into an $U B$ for $C_{i}$. To put this into practice we need to address the following issues: (1) how to select the basic cost expression $j$ from the products in order to build $C_{i j}$; and (2) how to compute an $U B$ for $C_{i j}$. In secs. 3.1 and 3.2 we discuss several methods for addressing the second issue. The first issue is discussed later in Sec. 3.4.

Let us first position our approach in the spectrum of related approaches [3]6. Solving $C_{i}$ using the techniques of [3] we obtain the $U B\left(\prod_{k=1}^{p_{i}} \hat{b}_{i k}(\bar{x})\right) \cdot \mathcal{N}$. Interestingly, this $U B$ can be explained using our novel view of Lemma 1, which is different from that of [3], as follows: we can consider $\hat{b}_{i j}(\bar{x}) \cdot \mathcal{N}$ as an $U B$ for $C_{i j}$, and then use it as in Lemma 1 to obtain $\left(\prod_{k=1}^{p_{i}} \hat{b}_{i k}(\bar{x})\right) \cdot \mathcal{N}$. Since, unlike [3], we focus on solving atomic $C R s$, we develop dedicated techniques (i.e., techniques that work only for atomic $C R s$ ) that are able to obtain an $U B$ far more precise than $\hat{b}_{i j}(\bar{x}) \cdot \mathcal{N}$ (we will usually eliminate the $\mathcal{N}$ factor). Solving $C_{i}$ using the techniques of [6] requires defining an $U B$ template to be used during the solving process. If $C_{i}$ does not admit an $U B$ that matches the supplied templates, then this technique will fail. Moreover, using arbitrary templates renders this approach impractical since it is based on the use of quantifier elimination procedure. Our techniques for solving atomic CRs are actually inspired by those 
of [6]. However, since we focus on a simpler form of $C R s$, we always use linear templates for which the quantifier elimination procedure is efficient. In summary, our approach uses [6] to precisely reason on the local cost of a single simple cost expression $b_{i j}$, and then uses 3 , to combine this local cost into an $U B$ for $C_{i}$.

To simplify our notation, in what follows, we assume a given atomic $C R D$ with $m$ equations of the form $\left\langle D(\bar{x})=e_{i}+\sum_{j=1}^{k_{i}} D\left(\bar{y}_{i j}\right), \varphi_{i}\right\rangle$, where $e_{1}=b$ is a basic cost expression, and $e_{i}=0$ for all $2 \leq i \leq m$. Note that the main equation of $D$ is the first one. We denote by $\bar{w}_{i}$ the set of variables in the $i$-th equation.

\subsection{The Tree-Sum Method}

We first explain this method for the case in which $b=$ nat $(l)$, and then we show how to extend it to handle any basic cost expression $b$. In many cases, in particular in examples that require amortised analysis, the sum of all instances of $b$ in any $T \in \operatorname{Trees}(D(\bar{v}))$ can be bounded by a linear expression. Thus, we seek an $U B$ for $D$ of the form $\alpha(\bar{x})=q_{0}+q_{1} \cdot x_{1}+\cdots+q_{n} \cdot x_{n}$, where $q_{i} \in \mathbb{Q}$. The way we search for $\alpha(\bar{x})$ is based on the use of universally quantified formulas as in [6]. We first define a verification condition which ensures that a given $\alpha(\bar{x})$ is a valid $U B$ for $D$. Then, using a quantifier elimination procedure, we turn this verification condition into a synthesis procedure that actually infers $\alpha(\bar{x})$.

Lemma 2. Let $\alpha(\bar{x})=q_{0}+q_{1} \cdot x_{1}+\cdots+q_{n} \cdot x_{n}$, and define:

$$
\begin{gathered}
\Psi_{1} \triangleq \forall \bar{w}_{1}: \varphi_{1} \rightarrow \operatorname{nat}(\alpha(\bar{x})) \geq \operatorname{nat}(l)+\sum_{j=1}^{k_{1}} \operatorname{nat}\left(\alpha\left(\bar{y}_{1 j}\right)\right) \\
\Psi_{2} \triangleq \bigwedge_{i=2}^{m} \forall \bar{w}_{i}: \varphi_{i} \rightarrow \operatorname{nat}(\alpha(\bar{x})) \geq \sum_{j=1}^{k_{i}} \operatorname{nat}\left(\alpha\left(\bar{y}_{i j}\right)\right) \\
\text { If } \Psi_{1} \wedge \Psi_{2} \text { is valid, then } \operatorname{nat}(\alpha(\bar{x})) \text { is an } \text { UB for the atomic CR } D .
\end{gathered}
$$

Intuitively, $\Psi_{1}$ requires that nat $(\alpha(\bar{x}))$ covers the cost of the main equation, i.e., it covers the local cost nat $(l)$ and the cost of the recursive calls. Similarly, $\Psi_{2}$ requires that nat $(\alpha(\bar{x}))$ covers the cost of the other equations (in this case the local cost is 0$)$. Our main interest is in inferring such $\alpha(\bar{x})$ rather than verifying the correctness of a given one. Turning the verification condition into an inference procedure can be done, using a quantifier elimination procedure, as follows:

1. we generate $\Psi_{1} \wedge \Psi_{2}$ using a template function $\alpha(\bar{x})$ in which $q_{0}, \ldots, q_{n}$ are variables, i.e., unknown;

2. we eliminate the universal quantifiers from $\Psi_{1} \wedge \Psi_{2}$. This results in a set of constraints $\Theta$ over the variables $q_{0}, \ldots, q_{n}$; and

3. any solution of $\Theta$ (i.e., values for $q_{0}, \ldots, q_{n}$ that satisfy $\Theta$ ) defines a valid $U B$ nat $(\alpha(\bar{x}))$. We simply pick a solution.

Note that if $\Theta$ is not satisfiable then there is no $\alpha(\bar{x})$ satisfying $\Psi_{1} \wedge \Psi_{2}$. In such case we say that the Tree-Sum method is not applicable for $D$. The main subtle point in the above inference procedure is how to eliminate the universal quantifiers, which is computationally expensive in general. However, since the formula $\Psi_{1} \wedge \Psi_{2}$ have a very specific form (almost linear), in Sec. 3.3 we show how this can be done efficiently. For now we just assume the existence of a procedure that implements steps (2) and (3) above. 
Example 5. Consider the $C R$ for $_{12}$, as defined in Ex. 4 and let $\alpha(e, d, s, i)=q_{0}+$ $q_{1} \cdot e+q_{2} \cdot d+q_{3} \cdot s+q_{4} \cdot i$. The corresponding $\Psi_{1}$ and $\Psi_{2}$ are:

$\Psi_{1} \triangleq \forall \bar{w}_{2}: \varphi_{2} \rightarrow \operatorname{nat}\left(q_{0}+q_{1} \cdot e+q_{2} \cdot d+q_{3} \cdot s+q_{4} \cdot i\right) \geq \operatorname{nat}(s)+\operatorname{nat}\left(q_{0}+q_{1} \cdot e+q_{2} \cdot d^{\prime}+q_{3} \cdot s^{\prime}+q_{4} \cdot i^{\prime}\right)$

$\Psi_{2} \triangleq \forall \bar{w}_{3}: \varphi_{3} \rightarrow \operatorname{nat}\left(q_{0}+q_{1} \cdot e+q_{2} \cdot d+q_{3} \cdot s+q_{4} \cdot i\right) \geq \operatorname{nat}\left(q_{0}+q_{1} \cdot e+q_{2} \cdot d+q_{3} \cdot s^{\prime}+q_{4} \cdot i^{\prime}\right)$

Solving $\Psi_{1} \wedge \Psi_{2}$, i.e finding values for $q_{0}, \ldots, q_{4}$, gets $q_{0}=-2, q_{1}=2, q_{2}=-1, q_{3}=2$, and $q_{4}=-2$, which means that for $_{12}^{+}(e, d, s, i)=\operatorname{nat}(2 \cdot s+2 \cdot e-2 \cdot i-d-2)$ is an $U B$ for $C R$ for $_{12}$. Then, to get an $U B$ for $C R$ for $_{1}$ we apply Lemma 1 which results in $f r_{1}^{+}(e, d, s, i)=2 \cdot \operatorname{nat}(2 \cdot s+2 \cdot e-2 \cdot i-d-2)$. Similarly, generating the formulas for for $_{21}$ and for $_{31}$ and solving them, we get the $U B s$ for $_{2}^{+}(e, d, s, i)=\operatorname{nat}(2 \cdot e-2 \cdot i)$ and $\operatorname{for}_{3}^{+}(e, d, s, i)=$ nat $(2 \cdot e-2 \cdot i)$. Finally, we can use Obs. 1 to add them in $f r^{+}(e, d, s, i)=2 \cdot \operatorname{nat}(2 \cdot s+2 \cdot e-2 \cdot i-d-2)+2 \cdot$ nat $(2 \cdot e-2 \cdot i)$ as $U B$ for for. Substituting this $U B$ in the equation of $a d d$ in Fig. 1. we get the expected linear bound $a d d^{+}(e, d, s)=2 \cdot \operatorname{nat}(2 \cdot s+2 \cdot e-d-2)+2 \cdot \operatorname{nat}(2 \cdot e)$ for method add.

Now we turn to the general case in which $b$ is an arbitrary basic cost expression, not necessarily nat $(l)$. In such cases, in addition to nat $(l), b$ can be of the form $\log _{a}(1+\operatorname{nat}(l))$ or $a^{\text {nat }(l)}-1$. Recall that when it is $q \in \mathbb{Q}^{+}$, we have implicitly assumed it was written as nat $(q)$. Note that in all cases $b$ has an embedded nat $(l)$ expression. Let $E$ be the $C R$ obtained from $D$ by replacing $b$ by its embedded nat $(l)$. Then the following lemma explains how to obtain an $U B$ for $D$ from that of $E$. Computing an $U B$ for $E$ is done as above.

Lemma 3. Let nat $(\alpha(\bar{x}))$ be an UB for $E$, and let

$$
D^{+}(\bar{x})= \begin{cases}\operatorname{nat}(\alpha(\bar{x})) & b=\operatorname{nat}(l) \\ 1.5 \cdot \operatorname{nat}(\alpha(\bar{x})) & b=\log _{a}(1+\operatorname{nat}(l)) \\ a^{\text {nat }(\alpha(\bar{x}))}-1 & b=a^{\text {nat }(l)}-1\end{cases}
$$

Then, $D^{+}(\bar{x})$ is an UB for $D$.

It is worth mentioning here the reason for which we use $a^{\text {nat }(l)}-1$ as a basic cost expression, instead of $a^{\text {nat }(l)}$. This allows precisely lifting the $U B$ of $E$ to an $U B$ of $D$ (in the last case of $D^{+}$), which is not possible when using $a^{\text {nat }(l)}$.

Example 6. Let us finish this section by trying to analyse the $C R$ qs using the Tree-Sum method. For $q s_{11}$, we first generate:

$$
\begin{aligned}
& \Psi_{1} \triangleq \forall \bar{w}_{1}: \psi_{1} \rightarrow \operatorname{nat}\left(q_{0}+q_{1} \cdot f+q_{2} \cdot t+q_{3} \cdot r\right) \geq \operatorname{nat}(t-f) \\
& \Psi_{2} \triangleq \forall \bar{w}_{2}: \psi_{2} \rightarrow \operatorname{nat}\left(q_{0}+q_{1} \cdot f+q_{2} \cdot t+q_{3} \cdot r\right) \geq \operatorname{nat}\left(q_{0}+q_{1} \cdot f+q_{2} \cdot m^{\prime}+q_{3} \cdot r\right)+ \\
& \operatorname{nat}\left(q_{0}+q_{1} \cdot m^{\prime \prime}+q_{2} \cdot t+q_{3} \cdot r\right)
\end{aligned}
$$

Solving $\Psi_{1} \wedge \Psi_{2}$ results in $q_{0}=0, q_{1}=-1, q_{2}=1$, and $q_{3}=0$. Thus, nat $(t-f)$ is an $U B$ for $q s_{11}$. Using Lemma 1 , we get $q s_{1}^{+}(f, t, r)=$ nat $(t-f)^{2}$. Solving $q s_{21}$ with the Tree-Sum method does not yield any result because the generated formula is not valid. This is expected since $q s_{21}$ does not have a linear bound. In Sec. 3.2 we develop further methods to handle such cases. 


\subsection{The Level-Sum Method}

In this section we describe our method for solving atomic $C R s$ that exhibit a divide and conquer like behaviour. As we have seen in Ex. 6, the Tree-Sum method fails to handle such examples. We first explain it for the case of $b=$ nat $(l)$, and then extend it to an arbitrary basic cost expression.

We start with some notation. Given an evaluation tree $T \in \operatorname{Trees}(D(\bar{v}))$, a node in $T$ is called primary if it is generated by the main equation. Note that the cost of all other nodes in $T$ is 0 . The primary-depth of a primary node is the number of primary nodes on the path from the root to that node (both included). The primary-depth of $T$, denoted by $\operatorname{pdepth}(T)$, is the maximum among the primary depths of all its primary nodes. The sum of (the cost of) all primary nodes of primary-depth $i$ is denoted by SumLevel $(T, i)$.

We say that nat $(\alpha(\bar{x}))$ is an UB on the primary-depth of $D$, if for any input $\bar{v}$ and $T \in \operatorname{Trees}(D(\bar{v}))$ we have nat $(\alpha(\bar{v})) \geq \operatorname{pdepth}(T)$. We say that it is an UB on the Level-Sum of $D$, if for any input $\bar{v}, T \in \operatorname{Trees}(D(\bar{v}))$, and $1 \leq i \leq \operatorname{pdepth}(T)$ we have nat $(\alpha(\bar{v})) \geq \operatorname{SumLevel}(T, i)$.

Lemma 4. Let nat $\left(\alpha_{1}(\bar{x})\right)$ and nat $\left(\alpha_{2}(\bar{x})\right)$ be UBs on the primary-depth and Level-Sum of $D$, respectively. Then, $\operatorname{nat}\left(\alpha_{1}(\bar{x})\right) \cdot \operatorname{nat}\left(\alpha_{2}(\bar{x})\right)$ is an UB for $D$.

The correctness of the above lemma follows from the fact that only primary nodes can have non-zero cost. Intuitively, the above lemma handles divide and conquer examples since, in such examples, the input is distributed between the recursive calls. Thus, the cost of all levels is similar and can be expressed as a linear function on the initial input. Moreover, using the primary-depth, instead of depth, allows ignoring those levels that do not contribute to the cost. Note that the above lemma also reduces the problem of solving $D$, to that of finding nat $\left(\alpha_{1}(\bar{x})\right)$ and nat $\left(\alpha_{2}(\bar{x})\right)$ that bound its primary-depth and Level-Sum. We start with bounding the primary-depth.

Lemma 5. Let $\alpha(\bar{x})=q_{0}+q_{1} \cdot x_{1}+\cdots+q_{n} \cdot x_{n}$, and define:

$$
\begin{gathered}
\Phi_{1} \triangleq \begin{cases}\forall \bar{w}_{1}: \varphi_{1} \quad \rightarrow \operatorname{nat}(\alpha(\bar{x})) \geq 1 & \text { if } k_{1}=0 \\
\bigwedge_{j=1}^{k_{1}} \forall \bar{w}_{1}: \varphi_{1} \rightarrow \operatorname{nat}(\alpha(\bar{x})) \geq 1+\operatorname{nat}\left(\alpha\left(\bar{y}_{1 j}\right)\right) & \text { if } k_{1} \geq 1\end{cases} \\
\Phi_{2} \triangleq \quad \bigwedge_{i=2}^{m} \bigwedge_{j=1}^{k_{i}} \forall \bar{w}_{i}: \varphi_{i} \rightarrow \operatorname{nat}(\alpha(\bar{x})) \geq \operatorname{nat}\left(\alpha\left(\bar{y}_{i j}\right)\right) \\
\text { If } \Phi_{1} \wedge \Phi_{2} \text { is valid, then } \operatorname{nat}(\alpha(\bar{x})) \text { is an } \mathrm{UB} \text { on the primary-depth of } D .
\end{gathered}
$$

Intuitively, the primary-depth corresponds to the number of applications of the main equation, in a sequence of recursive calls. This is reflected in $\Phi_{1}$ and $\Phi_{2}$ as follows. In $\Phi_{1}$, we treat applications of the main equation. If the main equation is non-recursive, i.e., $k_{1}=0$, then we require that nat $(\alpha(\bar{x}))$ covers that single application. In case it is recursive, i.e., $k_{1} \geq 1$, then we require that nat $(\alpha(\bar{x}))$ covers that application and further ones that might arise through each recursive call. In $\Phi_{2}$, we treat applications of other equations. In such case we require that nat $(\alpha(\bar{x}))$ covers applications of the main equation that might arise through each recursive call. Note that each recursive call is considered separately, since we count primary nodes in each path rather than the whole tree. 
It is worth noting that if we apply $\Phi_{1}$ to all equations instead of only the main one, then nat $(\alpha(\bar{x}))$ bounds the depth of any evaluation tree rather than the primary-depth. Similar techniques, based on inference of (linear) ranking functions, were used in 3 to bound the depth of the evaluation trees.

Example 7. Applying Lemma 5 to bound the primary-depth of $q s_{21}$ (of Ex. 4) results in $\Phi_{2}=$ true and $\Phi_{1}$ as the conjunction of the following formulas:

$$
\begin{aligned}
& \forall \bar{w}_{2}: \psi_{2} \rightarrow \operatorname{nat}\left(q_{0}+q_{1} \cdot f+q_{2} \cdot t+q_{3} \cdot r\right) \geq 1+\operatorname{nat}\left(q_{0}+q_{1} \cdot f+q_{2} \cdot m^{\prime}+q_{3} \cdot r\right) \\
& \forall \bar{w}_{2}: \quad \psi_{2} \rightarrow \operatorname{nat}\left(q_{0}+q_{1} \cdot f+q_{2} \cdot t+q_{3} \cdot r\right) \geq 1+\operatorname{nat}\left(q_{0}+q_{1} \cdot m^{\prime \prime}+q_{2} \cdot t+q_{3} \cdot r\right)
\end{aligned}
$$

Both originate from the recursive equation of $q s_{2}$. They respectively correspond to the first and second calls. Solving $\Phi_{1} \wedge \Phi_{2}$ results in $q_{0}=1, q_{1}=-1, q_{2}=1$, $q_{3}=0$, which induces the $U B$ nat $(t-f+1)$ on the primary-depth of $q s_{21}$.

Now we turn to bounding the Level-Sum of $D$.

Lemma 6. Let $\alpha(\bar{x})=q_{0}+q_{1} \cdot x_{1}+\cdots+q_{n} \cdot x_{n}$, and define:

$$
\begin{aligned}
& \Pi_{1} \triangleq \forall \bar{w}_{1}: \varphi_{1} \rightarrow \quad \operatorname{nat}(\alpha(\bar{x})) \geq \operatorname{nat}(l) \\
& \Pi_{2} \triangleq \bigwedge_{i=1}^{m} \forall \bar{w}_{i}: \varphi_{i} \rightarrow \quad \operatorname{nat}(\alpha(\bar{x})) \geq \sum_{j=1}^{k_{i}} \operatorname{nat}\left(\alpha\left(\bar{y}_{i j}\right)\right)
\end{aligned}
$$

If $\Pi_{1} \wedge \Pi_{2}$ is valid, then $\operatorname{nat}(\alpha(\bar{x}))$ is an UB on the Level-Sum of $D$.

Intuitively, $\Pi_{1}$ requires that nat $(\alpha(\bar{x}))$ covers the local cost of the main equation at any level, and $\Pi_{2}$ requires that it also covers the next level. Combining these conditions, and applying inductive reasoning, one can conclude that $\operatorname{nat}(\alpha(\bar{x}))$ is actually an $U B$ on the Level-Sum of $D$.

Example 8. Consider again $q s_{21}$ (of Ex. 4). Its corresponding formulas are:

$$
\begin{aligned}
& \Pi_{1} \triangleq \forall \bar{w}_{2}: \psi_{2} \rightarrow \operatorname{nat}\left(q_{0}+q_{1} \cdot f+q_{2} \cdot t+q_{3} \cdot r\right) \geq \operatorname{nat}(t-f) \\
& \Pi_{2} \triangleq \forall \bar{w}_{2}: \psi_{2} \rightarrow \operatorname{nat}\left(q_{0}+q_{1} \cdot f+q_{2} \cdot t+q_{3} \cdot r\right) \geq \operatorname{nat}\left(q_{0}+q_{1} \cdot f+q_{2} \cdot m^{\prime}+q_{3} \cdot r\right)+ \\
& \text { nat }\left(q_{0}+q_{1} \cdot m^{\prime \prime}+q_{2} \cdot t+q_{3} \cdot r\right)
\end{aligned}
$$

Solving $\Pi_{1} \wedge \Pi_{2}$ results in $q_{0}=0, q_{1}=-1, q_{2}=1, q_{3}=0$. This induces the bound nat $(t-f)$ on the Level-Sum. Combining this bound with that in Ex. 7 , on the primary depth, we obtain nat $(t-f) \cdot \operatorname{nat}(t-f+1)$ as an $U B$ for $q s_{21}$, which is also an $U B$ for $q s_{2}$. Combining this, using Obs. 1, with the bound of $q s_{1}$ computed in Ex. 66, we get $q s^{+}(f, t, r)=\operatorname{nat}(t-f) \cdot \operatorname{nat}(t-f+1)+\operatorname{nat}(t-f) \cdot \operatorname{nat}(t-f)$. Substituting this $U B$ in the equation of qsort in Fig. 1 we obtain qsort $^{+}(s, r)=\operatorname{nat}(s-1)$. nat $(s)+\operatorname{nat}(s-1) \cdot \operatorname{nat}(s-1)$, which is the expected bound for method qsort.

Turning the verification condition to inference procedure, both in Lemma 5 and Lemma 6, is done as we explained in Sec. 3.1. Handling the general case in which $b$ is an arbitrary basic cost expression, is done exactly as the case of Tree-Sum (see Lemma 3). Note that this affects only the $U B$ on the Level-Sum.

Finally, we note that [3] proposed a technique for solving CRs with a divide and conquer behaviour, however, it is limited to cases in which: (1) the cost of all levels is non-increasing; and (2) the cost expression of each equation is linear. Note that, $C R q s_{1}$, for example, does not satisfy both conditions. 


\subsection{Solving the Universally Quantified Formulas}

In this section we describe how we solve the universally quantified formulas of Lemma 2, Lemma 5, and Lemma 6. Namely, starting from a template linear function $\alpha(\bar{x})=q_{0}+q_{1} \cdot x_{1}+\ldots+q_{n} \cdot x_{n}$, we find rational values for $q_{0}, \ldots, q_{n}$ for which the corresponding formula is valid. Note that our formulas are conjunctions of universally quantified formulas of the following form:

$$
\forall \bar{w}: \varphi \rightarrow \operatorname{nat}\left(l_{0}\right) \geq q+\operatorname{nat}\left(l_{1}\right)+\ldots+\operatorname{nat}\left(l_{n}\right)
$$

where $\varphi$ defines a closed polyhedron, $q \in\{0,1\}$, and each $l_{i}$ is either a linear function over $\bar{w}$, or a template function $\alpha(\bar{x})=q_{0}+q_{1} \cdot x_{1}+\cdots+q_{n} \cdot x_{n}$ such that $\bar{x} \subseteq \bar{w}$ and $q_{i} \notin \bar{w}$ (i.e., each $q_{i}$ is existentially quantified). Our goal is to solve these formulas using linear programming (LP) techniques.

Consider a formula as in (1), but without the nat-expressions, i.e., of the form $\forall \bar{w}: \varphi \rightarrow l_{0} \geq q+l_{1}+\ldots+l_{n}$. It is known that there is a complete algorithm, based on the use of LP [8, able to solve such a formula. Our aim is to transform formulas as (1) to a nat-free as above, and then solve them using this algorithm. Recall that nat $\left(l_{i}\right)=\max \left\{l_{i}, 0\right\}$. This means that nat $\left(l_{i}\right)$ can be eliminated by explicitly considering the cases for $l_{i} \geq 0$ and $l_{i} \leq 0$ (we use $l_{i} \geq 0$ and not $l_{i}>0$ since in LP constraints must be non-strict). For example, eliminating nat $\left(l_{0}\right)$ can be done by rewriting (1) as:

$$
\begin{aligned}
& \forall \bar{w}: \varphi \wedge l_{0} \geq 0 \rightarrow l_{0} \geq q+\operatorname{nat}\left(l_{1}\right)+\ldots+\operatorname{nat}\left(l_{n}\right) \wedge \\
& \forall \bar{w}: \varphi \wedge l_{0} \leq 0 \rightarrow 0 \geq q+\operatorname{nat}\left(l_{1}\right)+\ldots+\operatorname{nat}\left(l_{n}\right)
\end{aligned}
$$

This process can be applied iteratively to eliminate each nat $\left(l_{i}\right)$. There is still one problem that prevents us from directly applying the LP techniques: when $l_{i}$ is a template function, the constraints $l_{0} \geq 0$ and $l_{0} \leq 0$ are not linear. To overcome this problem, assuming that eliminating the nat-expression results in a formula $\xi$, we generated $\xi^{\prime}$ be the by simply removing all non-linear constraints from $\xi$. Since all non-linear constraints in $\xi$ appear in the left-hand sides of the implications, we observe that $\xi^{\prime} \rightarrow \xi$. This means that we can solve $\xi^{\prime}$, using the LP based algorithm, instead of $\xi$. Although we scarify completeness, this approach performs well in practice as demonstrated by our experiments.

\subsection{Concluding Remarks}

Let us conclude this section describing how all pieces, that have been described so far, connects together to infer an $U B$ for $C$.

Solving CR $C$. This is as done according to the following steps: (1) generating the sparse $C R s C_{1}, \ldots, C_{t}$ of $C$; (2) solving each $C_{i}$ into an $U B$ as described below; and (3) combining these $U B s$, as in Obs. 11 into an $U B$ for $C$.

Solving a sparse CR $C_{i}$. This step requires solving, using the methods described in secs. 3.1 and 3.2 , one $C_{i j}$ of the corresponding atomic $C R$ which might fail for some $j$ and succeed for some others. We iterate over all possible $j=1, \ldots, p_{i}$, and if all fail then we solve $C_{i}$ using the approach of [3].

Solving an atomic CR $C_{i j}$. This is done by trying the methods of secs. 3.1 and 3.2 , in this order. Note that in [1] we describe some additional methods. 
Table 1. Experimental comparison with PUBS [3]. The times on the right (in secs) correspond to analysing a $C R$ that connects all benchmarks together (see Sec. 4 ).

\begin{tabular}{|c|c|c|}
\hline Entry & $\mathcal{O}(u b)-$ new & $\mathcal{O}(u b)-$ PUBS \\
\hline $\operatorname{add}(a, b, c)$ & $\operatorname{nat}(a)+\operatorname{nat}(2 a-b+2 c)$ & $\operatorname{nat}(a) \cdot \operatorname{nat}(a+c)$ \\
\hline qsort $(a, b, c)$ & $\operatorname{nat}(a)^{2}$ & $2^{\text {nat }(a)} \cdot(\operatorname{nat}(a)+\operatorname{nat}(b))$ \\
\hline $\operatorname{sum}(\mathrm{a})$ & $\operatorname{nat}(a)$ & $2^{\text {nat }(a)} \cdot \operatorname{nat}(a)$ \\
\hline $\operatorname{dac}(a, b)$ & $\operatorname{nat}(a)^{2}+\operatorname{nat}(a-b)$ & $2^{\text {nat }(b)} \cdot \operatorname{nat}(a)$ \\
\hline $\log (a, b)$ & $\operatorname{nat}(b)+\operatorname{nat}(a) \cdot \log (\operatorname{nat}(b))$ & $\operatorname{nat}(b) \cdot \operatorname{nat}(a)$ \\
\hline once $(\mathrm{a}, \mathrm{b})$ & $\operatorname{nat}(a)+\operatorname{nat}(b)$ & $\operatorname{nat}(b) \cdot \operatorname{nat}(a)$ \\
\hline twice $(a, b)$ & $\operatorname{nat}(a)+\operatorname{nat}(b)$ & $\operatorname{nat}(b) \cdot \operatorname{nat}(a)$ \\
\hline full $(a, b)$ & $\operatorname{nat}(a) \cdot \operatorname{nat}(b)$ & $\operatorname{nat}(a) \cdot \operatorname{nat}(b)^{2}$ \\
\hline eratos $(\mathrm{a})$ & $\operatorname{nat}(a)$ & $\operatorname{nat}(a)^{2}$ \\
\hline peak $(\mathrm{a})$ & $\operatorname{nat}(a)$ & $\operatorname{nat}(a) \cdot \log (\operatorname{nat}(a))$ \\
\hline $\operatorname{stack}(a, b, c)$ & $\operatorname{nat}(b) \cdot \operatorname{nat}(c)+\operatorname{nat}(b)^{2}$ & $\operatorname{nat}(c) \cdot \operatorname{nat}(b)^{2}$ \\
\hline $\operatorname{rotate}(\mathrm{a}, \mathrm{b})$ & $\operatorname{nat}(a)+\operatorname{nat}(b)+\operatorname{nat}(a-b)$ & $\operatorname{nat}(a) \cdot \operatorname{nat}(a-b)$ \\
\hline $\operatorname{maxsum}(a, b)$ & $\operatorname{nat}(b) \cdot \log (\operatorname{nat}(b))$ & $\operatorname{nat}(b)^{2}$ \\
\hline mayor(a) & $\operatorname{nat}(a) \cdot \log (\operatorname{nat}(a))$ & $\operatorname{nat}(a) \cdot \log (\operatorname{nat}(a))$ \\
\hline $\operatorname{msort}(\mathrm{a}, \mathrm{b}, \mathrm{c}, \mathrm{d})$ & $\operatorname{nat}(d-c) \cdot \log (\operatorname{nat}(d-c))$ & $\operatorname{nat}(d-c)^{2}$ \\
\hline mergexp(a) & $\operatorname{nat}(a)$ & $\operatorname{nat}(a) \cdot \log (\operatorname{nat}(a))$ \\
\hline enque $(a, b, c, d)$ & nat $(c+d)+\operatorname{nat}(a+c)$ & nat $(c+d) \cdot \operatorname{nat}(a+c)$ \\
\hline deque $(a, b, c)$ & $\operatorname{nat}(a)+\operatorname{nat}(c)$ & $\operatorname{nat}(c) \cdot \operatorname{nat}(a)$ \\
\hline infinity(a) & $\operatorname{nat}(a)$ & Failed: No RF \\
\hline
\end{tabular}

\begin{tabular}{|r|r|r|r|}
\hline $\mathbf{E q}$ & $\mathbf{T}_{n}$ & $\mathbf{T}_{p}$ & $\mathbf{O v}$ \\
\hline 11 & 0.15 & 0.11 & 1.34 \\
\hline 28 & 0.61 & 0.27 & 2.26 \\
\hline 36 & 0.88 & 0.33 & 2.63 \\
\hline 45 & 1.24 & 0.40 & 3.13 \\
\hline 54 & 1.71 & 0.47 & 3.63 \\
\hline 62 & 1.98 & 0.57 & 3.47 \\
\hline 70 & 2.29 & 0.69 & 3.33 \\
\hline 78 & 2.74 & 0.84 & 3.26 \\
\hline 91 & 3.16 & 0.94 & 3.37 \\
\hline 96 & 3.43 & 1.01 & 3.38 \\
\hline 107 & 3.95 & 1.19 & 3.32 \\
\hline 120 & 4.84 & 1.62 & 2.99 \\
\hline 138 & 7.67 & 2.12 & 3.62 \\
\hline 163 & 13.21 & 3.20 & 4.13 \\
\hline 173 & 13.72 & 3.81 & 3.60 \\
\hline 187 & 14.65 & 4.02 & 3.65 \\
\hline 199 & 16.34 & 4.68 & 3.49 \\
\hline 208 & 17.40 & 4.91 & 3.55 \\
\hline 219 & 18.38 & 5.07 & 3.63 \\
\hline
\end{tabular}

\section{Implementation and Experiments}

We have implemented our techniques as an extension of PUBS $[\underline{3}$, the solver used in Costa 4 for solving $C R s$ generated from JAVA programs. This allows us to evaluate our approach directly on JAVA programs. We evaluate accuracy and scalability on a set of benchmarks that we collected from related literature, or were written to demonstrate some powerful features of our approach. Although the programs are not large, they exhibit challenging behaviour for cost analysis. The benchmarks and the implementation are available online [1].

In Table 1 we evaluate the accuracy of our approach by comparing it to PUBS [3. We applied both approaches on each benchmark using a cost model that measure memory consumption or visits to an specific program point (depending on what was more interesting for each benchmark). Each line includes (from left to right) the entry method and its parameters, the $U B$ inferred by our approach and the $U B$ inferred by PUBS. For readability, bounds are given in asymptotic form 2. In all examples our approach obtains $U B s$ that are asymptotically more accurate than those obtained by PUBS. Moreover, our UBs approach obtains precise asymptotic $U B s$, i.e., they exactly reflect the actual cost.

To analyse scalability, we have merged all our benchmarks into a single program as follows: the benchmark in row $i$ was modified to include a call (in one of its loops) to the program at row $i-1$. This means that the $i$-th benchmark executes at least $i$ nested loops. The runtime (in seconds) of analysing each such (modifed) benchmark is depicted in columns $\mathbf{T}_{n}$ (current approach) and 
$\mathbf{T}_{p}$ (PUBs) of Table 1. Columns Eq and $\mathbf{O v}$ are, respectively, the total number of equations and the overhead $\left(\mathbf{T}_{n} / \mathbf{T}_{p}\right)$ introduced by our approach.

We have also compared our approach to [6]. For all benchmarks of Table 1 . it did not obtain an $U B$ within the one minute time limit. This is expected since it is based on a general procedure for real quantifier elimination.

\section{Conclusions and Related Work}

In this paper we have developed a novel approach for solving $C R s$ into precise closed-form UBs. It is based on the idea of dividing the basic cost expressions of a given $C R C$ into two parts: (a) those for which we employ precise reasoning to track their behaviour along the execution; and (b) those for which we simply use their worst case behavior. Then, we show how such different bounds can be combined into an $U B$ for $C$. For part (b) we rely on existing techniques [4] to maximise cost expressions. For part (a) we first model the contribution of the corresponding cost expressions using universally quantified formulas, and then, a precise $U B$ on their costs can be obtain by eliminating the universal quantifiers. Note that while quantifier elimination is a very expensive procedure in general, in our case, since the formulas are of a very specific form, they can be solved efficiently. Our method has been implemented within Costa [4, and preliminary experiments demonstrate its superiority on previous methods for solving $C R s$.

Related work. The most related works to ours are [4] which aim at solving CRs into closed-form UBs. In Sec. 4 we have seen that, in practice, our approach is more precise than 4 and more efficient than 6. Detailed discussion on similarities and differences is provided along Sec. 3 . Note that although the method described so far is usually more precise than [3, as we have seen in Sec. 4 , there are some examples for which the use of the last case of Lemma 3 causes a loss of precision. E.g., replacing nat $(s)$ by $2^{\text {nat }(s)}$ in for ${ }_{12}$ of Ex. 4, the approach of [3] obtains nat $(e-i) \cdot 2^{\text {nat }(s+e-1)}$ while we obtain $2^{\text {nat }(2(s+e-i-1)-d)}$. In [5], the techniques of [4] were improved to handle cases in which the cost can be modeled with arithmetic or geometric sequences. This approach is complementary to ours, in the sense that it cannot handle our benchmarks and we cannot handle some of their examples (when basic cost expressions require non-linear bounds).

There are some works that aim at inferring loop bounds on the visits to a given program point [14|19]. They are mostly related to our Lemma 5. These approaches are not limited to linear bounds, however, they cannot handle recursive programs with more than one recursive call. Our techniques can benefit from these approaches when each cost equation has at most one recursive call. Cost analysis techniques that are based on amortised analysis [15]16], could, in principle, handle some of our examples when the bounds are polynomial, and the data are over the non-negative integers. Solving $C R s$ using template functions and real quantifier elimination has been considered before in [7. Finally, several cost analysis frameworks 911] that are based on generating CRs can benefit from our advances in solving CRs. 
Acknowledgements This work was funded partially by the projects FP7-ICT610582, TIN2008-05624, TIN2012-38137, PRI-AIBDE-2011-0900 and S2009TIC1465. Diego Esteban Alonso-Blas is supported by the $\mathrm{PhD}$ scholarship program of the Complutense University.

\section{References}

1. Companion Web-Page. http://costa.ls.fi.upm.es/amor/

2. E. Albert, P. Arenas, D. Alonso, S. Genaim, and G. Puebla. Asymptotic Resource Usage Bounds. In APLAS, volume 5904 of $L N C S$, pages 294-310. Springer, 2009.

3. E. Albert, P. Arenas, S. Genaim, and G. Puebla. Closed-Form Upper Bounds in Static Cost Analysis. J. Autom. Reasoning, 46(2):161-203, 2011.

4. E. Albert, P. Arenas, S. Genaim, G. Puebla, and D. Zanardini. Cost Analysis of Object-Oriented Bytecode Programs. Theor. Comput. Sci., 413(1):142-159, 2012.

5. E. Albert, S. Genaim, and A.N. Masud. On the Inference of Resource Usage Upper and Lower Bounds. ACM Trans. Comput. Log. To appear.

6. D. E. Alonso-Blas and S. Genaim. On the Limits of the Classical Approach to Cost Analysis. In $S A S$, volume 7460 of $L N C S$, pages 405-421. Springer, 2012.

7. H. Anderson, S.C Khoo, S. Andrei, and B. Luca. Calculating Polynomial Runtime Properties. In APLAS, volume 3780 of $L N C S$, pages 230-246. Springer, 2005.

8. R. Bagnara, F. Mesnard, A. Pescetti, and E. Zaffanella. A new look at the automatic synthesis of linear ranking functions. Inf. Comput., 215:47-67, 2012.

9. R. Benzinger. Automated higher-order complexity analysis. Theor. Comput. Sci., 318(1-2):79-103, 2004.

10. N. Danner, J. Paykin, and J.S. Royer. A Static Cost Analysis for a Higher-Order Language. In PLPV, pages 25-34. ACM, 2013.

11. S. K. Debray and N. Lin. Cost Analysis of Logic Programs. ACM Trans. Program. Lang. Syst., 15(5):826-875, 1993.

12. B. Grobauer. Cost Recurrences for DML Programs. In ICFP, pages 253-264. ACM, 2001.

13. S. Gulwani, J.K. Mehra, and T.M. Chilimbi. SPEED: Precise and Efficient Static Estimation of Program Computational Complexity. In POPL, pages 127-139. ACM, 2009.

14. S. Gulwani and F. Zuleger. The Reachability-Bound Problem. In PLDI, pages 292-304. ACM, 2010.

15. J. Hoffmann, , K. Aehlig, and M. Hofmannn. Multivariate Amortized Resource Analysis. ACM Trans. Program. Lang. Syst., 34(3):14:1-14:62, 2012.

16. H. R. Simões, P. B. Vasconcelos, M. Florido, S. Jost, and K. Hammond. Automatic Amortised Analysis of Dynamic Memory Allocation for Lazy Functional Programs. In ICFP, pages 165-176. ACM, 2012.

17. P. B. Vasconcelos and K. Hammond. Inferring Cost Equations for Recursive, Polymorphic and Higher-Order Functional Programs. In IFL, volume 3145 of LNCS, pages 86-101. Springer, 2003.

18. B. Wegbreit. Mechanical Program Analysis. Commun. ACM, 18(9):528-539, 1975.

19. F. Zuleger, S. Gulwani, M. Sinn, and H. Veith. Bound Analysis of Imperative Programs with the Size-Change Abstraction. In $S A S$, volume 6887 of $L N C S$, pages 280-297. Springer, 2011. 